\title{
Hill-Type Formula and Krein-Type Trace Formula for Hamiltonian Systems
}

\author{
Xijun $\mathrm{Hu}^{1, *}$, Yuwei $\mathrm{Ou}^{2}$, Penghui Wang ${ }^{1}$ and Hao $\mathrm{Zhu}^{3}$ \\ 1 School of Mathematics, Shandong University, Jinan, Shandong 250100, China \\ 2 School of Mathematics (Zhuhai), Sun Yat-Sen University, Zhuhai, \\ Guangdong 519082, China \\ ${ }^{3}$ Department of Mathematics, Nanjing University, Nanjing, Jiangsu 210093, China
}

Received 30 August 2020; Accepted (in revised version) 16 October 2020

Dedicated to Prof. Paul H. Rabinowitz with admiration on the occasion of his 80th birthday

\begin{abstract}
In this paper, we give a survey on the Hill-type formula and its applications. Moreover, we generalize the Hill-type formula for linear Hamiltonian systems and Sturm-Liouville systems with any self-adjoint boundary conditions, which include the standard Neumann, Dirichlet and periodic boundary conditions. The Hill-type formula connects the infinite determinant of the Hessian of the action functional with the determinant of matrices which depend on the monodromy matrix and boundary conditions. Further, based on the Hill-type formula, we derive the Krein-type trace formula. As applications, we give nontrivial estimations for the eigenvalue problem and the relative Morse index.
\end{abstract}

Key Words: Hill-type formula, trace formula, conditional Fredholm determinant, relative Morse index.

AMS Subject Classifications: 34B30, 34L15, 34B09, 37C75, 70H14

\section{Introduction}

The study of Hill-type formula begins with the original work of Hill [10] in 1877. In his study of the motion of lunar perigee, Hill considered the following equation:

$$
\ddot{x}(t)+\theta(t) x(t)=0,
$$

where

$$
\theta(t)=\sum_{j \in \mathbb{Z}} \theta_{j} e^{2 j \sqrt{-1} t} \quad \text { with } \quad \theta_{0} \neq 0
$$

\footnotetext{
*Corresponding author. Email addresses: xjhu@sdu.edu.cn (X. J. Hu), ouyw3@mail.sysu.edu.cn (Y. W. Ou), phwang@sdu .edu.cn (P. H. Wang), haozhu@nju.edu.cn (H. Zhu) 
is a real $\pi$-periodic function. Let $\gamma(t)$ be the fundamental solution of the associated first order system of (1.1), that is,

$$
\dot{\gamma}(t)=\left(\begin{array}{cc}
0 & -\theta(t) \\
1 & 0
\end{array}\right) \gamma(t), \quad \gamma(0)=I_{2} .
$$

Suppose

$$
\rho=e^{c \sqrt{-1} \pi}, \quad \rho^{-1}=e^{-c \sqrt{-1} \pi},
$$

are the eigenvalues of the monodromy matrix $\gamma(\pi)$. In order to compute $c$, Hill obtained the following formula which connects the infinite determinant, corresponding to the differential operator, and the characteristic polynomial:

$$
\frac{\sin ^{2}\left(\frac{\pi}{2} c\right)}{\sin ^{2}\left(\frac{\pi}{2} \theta_{0}\right)}=\operatorname{det}\left[\left(-\frac{d^{2}}{d t^{2}}-\theta_{0}\right)^{-1}\left(-\frac{d^{2}}{d t^{2}}-\theta\right)\right],
$$

where the right hand side of (1.2) is the Fredholm determinant. We should point out that the right hand side of the original formula of Hill [10] is a determinant of an infinite matrix. In [10], Hill did not prove the convergence of the infinite determinant, and the convergence was proved by Poincaré [24]. The Hill-type formula for a periodic solution of Lagrangian system on manifold was given by Bolotin [2]. In [3], Bolotin and Treschev studied the Hill-type formula for both continuous and discrete Lagrangian systems with Legendre convexity condition. For the periodic solution of ODE, the Hill-type formula was given by Denk [6]. Please refer more works for Lagrangian systems at [5,7,16,21]. As the beginning of a series of work, Hu and Wang [15] introduced the conditional Fredholm determinant and sucessfully generalized the Hill-type formula to Hamiltonian systems with S-periodic boundary conditions. Together with $\mathrm{Ou}$, they obtained the Hill-type formula for Hamiltonian systems with Lagrangian boundary conditions [13]. For the Sturm-Liouville systems, they derived the Hill-type formula with $S$-periodic boundary conditions in [12] and with Lagrangian boundary conditions in [17]. By Taylor expansion of the parameterized Hill-type formula, they also derived the Krein-type trace formula $[12,13,17]$. For the non-self-adjoint version of the Hill-type formula, we refer the readers to [16].

The goal of this paper is to derive the Hill-type formula and the Krein-type trace formula for Hamiltonian systems and Sturm-Liouville systems with any self-adjoint boundary conditions, which cover $S$-periodic boundary conditions and Lagrangian boundary conditions. The linear Hamiltonian system takes the form

$$
\dot{z}(t)=J_{n}(B(t)+\lambda D(t)) z(t),
$$

where $B, D \in C([0, T] ; \mathcal{S}(2 n))$, and

$$
J_{n}=\left[\begin{array}{cc}
0 & -I_{n} \\
I_{n} & 0
\end{array}\right]
$$


Here, we denote by $\mathcal{M}(2 n)$ and $\mathcal{S}(2 n)$ the set of $2 n \times 2 n$ matrices and real symmetric matrices, respectively. $B$ and $D$ can be considered as bounded operators on $\mathcal{H}:=$ $L^{2}\left([0, T] ; \mathbb{C}^{2 n}\right)$, defined by $(B x)(t)=B(t) x(t)$ and $(D x)(t)=D(t) x(t)$. Let $\gamma_{\lambda}(t)$ be the fundamental solution of (1.3), that is,

$$
\dot{\gamma}_{\lambda}(t)=J_{n}(B(t)+\lambda D(t)) \gamma_{\lambda}(t), \quad \gamma_{\lambda}(0)=I_{2 n} .
$$

It is well known that

$$
\gamma_{\lambda}(t) \in \operatorname{Sp}(2 n):=\left\{M \in G L\left(\mathbb{R}^{2 n}\right) \mid M^{T} J_{n} M=J_{n}\right\}
$$

for any $t \in[0, T]$.

The self-adjoint boundary condition can be described by Lagrangian subspaces. More precisely, the standard symplectic structure $\omega_{n}(x, y)$ on $\mathbb{C}^{2 n}$ is defined by

$$
\omega_{n}(x, y)=\left\langle J_{n} x, y\right\rangle
$$

where $\langle\cdot, \cdot\rangle$ is the standard Hermitian inner product. A Lagrangian subspace $V$ of $\left(\mathbb{C}^{2 n}, \omega_{n}\right)$ is an isotropic subspace of dimension $n$, that is, for any $x, y \in V, \omega_{n}(x, y)=0$. Denote by $\operatorname{Lag}\left(\mathbb{C}^{2 n}, \omega_{n}\right)$ the set of Lagrangian subspaces of $\mathbb{C}^{2 n}$. It is well known that $\operatorname{Lag}\left(\mathbb{C}^{2 n}, \omega_{n}\right)$ is homeomorphic to the unitary group $\mathbb{U}(n)$. Let

$$
(\mathcal{V}, \Omega):=\left(\mathbb{C}^{2 n} \oplus \mathbb{C}^{2 n},-\omega_{n} \oplus \omega_{n}\right),
$$

which is $4 n$-dimensional symplectic space. As above, we denote the set of $2 n$ dimensional Lagrangian subspaces by $\operatorname{Lag}(\mathcal{V}, \Omega)$. Then any self-adjoint boundary condition can be written as

$$
\left[\begin{array}{l}
z(0) \\
z(T)
\end{array}\right] \in \Lambda
$$

where $\Lambda \in \operatorname{Lag}(\mathcal{V}, \Omega)$.

A frame for $\Lambda$ is a linear map $Z: \mathbb{C}^{2 n} \rightarrow \mathbb{C}^{4 n}$, whose image is $\Lambda$. Assume the frame of $\Lambda$ is of the form $\left[\begin{array}{l}X \\ Y\end{array}\right]$, where $X$ and $Y$ are $2 n \times 2 n$ matrices and satisfy

$$
X^{*} J_{n} X=Y^{*} J_{n} Y
$$

We will explain some important boundary conditions. Let

$$
\operatorname{Sp}_{\mathbb{C}}(2 n):=\left\{M \in G L\left(\mathbb{C}^{2 n}\right) \mid M^{*} J_{n} M=J_{n}\right\}
$$

be the space of complex symplectic matrices. The $M$-periodic orbit is a solution of (1.3), which satisfies $z(0)=M z(T)$ for some $M \in \operatorname{Sp}_{\mathbb{C}}(2 n)$. In this case, a frame of the boundary condition $\Lambda_{0}$ is $\left[\begin{array}{l}M \\ I_{2 n}\end{array}\right]$. Obviously, let $M=I_{2 n}$, we get the standard periodic boundary conditions. An important case is $M \in \operatorname{Sp}(2 n) \cap \mathrm{O}(2 n)$, which appears 
for symmetry periodic orbits. Another important case is the separated boundary conditions, which include the Dirichlet and Neumann boundary conditions. More precisely, let $z(0) \in V_{0}, z(T) \in V_{1}$, then $\Lambda_{0}=V_{0} \oplus V_{1}$, where $V_{0}, V_{1} \in \operatorname{Lag}\left(\mathbb{C}^{2 n}, \omega_{n}\right)$. Both the above boundary conditions naturally appear in the $n$-body problem.

We define the operator

$$
\left.A\right|_{E_{\Lambda}}=-J_{n} \frac{d}{d t} \text { on } \mathcal{H}
$$

with domain

$$
E_{\Lambda}=\left\{z \in W^{1,2}\left([0, T] ; \mathbb{C}^{2 n}\right) \mid\left[\begin{array}{l}
z(0) \\
z(T)
\end{array}\right] \in \Lambda\right\} .
$$

It is standard that $\left.A\right|_{E_{\Lambda}}$ is a self-adjoint operator and has compact resolvent. Obviously, the property of $\left.A\right|_{E_{\Lambda}}$ depends on its domain $E_{\Lambda}$ heavily and the choice of $E_{\Lambda}$ is based on the boundary conditions $\Lambda$. In what follows, we will write $A=\left.A\right|_{E_{\Lambda}}$ if there is no confusion.

It is well known that for $\lambda \in \rho(A)$, the resolvent $(A-\lambda)^{-1}$ is not a trace class operator, but a Hilbert-Schmidt operator. Assume that $A-B$ is non-degenerate throughout this paper, then we set

$$
\mathcal{F}\left(B, D ; E_{\Lambda}\right)=D(A-B)^{-1},
$$

which will be written as $\mathcal{F}(B, D)$ for short without confusion. Throughout this paper, we use $I$ to denote the identity on a Hilbert space. Since $\mathcal{F}(B, D)$ is not necessarily a trace class operator, the Fredholm determinant $\operatorname{det}(I-\mathcal{F}(B, D))$ is not well-defined. Instead we will use the definition of conditional Fredholm determinant, which was introduced in [15], see Section 2 for details. Another definition of the infinite dimensional determinant based on zeta function is referred to $[9,26]$.

Recall that $\left(X^{T}, Y^{T}\right)^{T}$ is a frame of $\Lambda$ in (1.4). Then we have the following Hill-type formula for Hamiltonian system (1.3)-(1.4).

Theorem 1.1. Assume that $A-B$ is non-degenerate, then

$$
\operatorname{det}(I-\mathcal{F}(B, D))=\operatorname{det}\left(\gamma_{1}(T) X-Y\right) \cdot \operatorname{det}\left(\gamma_{0}(T) X-Y\right)^{-1},
$$

where the left hand side is the conditional Fredholm determinant, and the right hand side is independent of the choice of the frame $\left(X^{T}, Y^{T}\right)^{T}$.

Remark 1.1. The conditional Fredholm determinant $\operatorname{det}(I-\mathcal{F}(B, D))$ depends on the projections $\left\{P_{N}\right\}$. Here, the choice of the projections $\left\{P_{N}\right\}$ is different from those in (1.9)(1.10). In fact, $\left\{P_{N}\right\}$ are chosen as a sequence of eigen-projections of the operator $-J_{n} \frac{d}{d t}$ associated with original S-periodic boundary condition in (1.9) and Lagrangian boundary condition in (1.10). We choose $\left\{P_{N}\right\}$ as $\{2,4\}$-components of $\left\{\hat{P}_{N}\right\}$, which is a sequence of eigen-projections of the operator $-J_{2 n} \frac{d}{d t}$ associated with the constructed Lagrangian boundary condition (3.3). 
Remark 1.2. Assume that $A-B$ is non-degenerate. Let $S \in \operatorname{Sp}(2 n) \cap \mathrm{O}(2 n)$. Then the following Hill-type formula for $S$-periodic boundary conditions was obtained in [15]:

$$
\operatorname{det}\left(I-\mathcal{F}\left(B, D ; E_{S}\right)\right)=\operatorname{det}\left(S \gamma_{1}(T)-I\right) \cdot \operatorname{det}\left(S \gamma_{0}(T)-I\right)^{-1}
$$

Let $Z_{0}, Z_{1}$ be frames of $\Lambda_{0}, \Lambda_{1}$. It is evident that $\gamma_{\lambda}(T) Z_{0}$ is a frame of $\gamma_{\lambda}(T) \Lambda_{0}$ and $\left(\gamma_{\lambda}(T) Z_{0}, Z_{1}\right)$ is a $2 n \times 2 n$ matrix. Then the following Hill-type formula for the real Lagrangian boundary conditions was obtained in [13]:

$$
\operatorname{det}\left(I-\mathcal{F}\left(B, D ; E_{\Lambda_{0}, \Lambda_{1}}\right)\right)=\operatorname{det}\left(\gamma_{1}(T) Z_{0}, Z_{1}\right) \cdot \operatorname{det}\left(\gamma_{0}(T) Z_{0}, Z_{1}\right)^{-1} .
$$

Here, the left hand side of (1.9)-(1.10) is the conditional Fredholm determinant, and the right hand side of (1.10) is independent of the choice of the frames $Z_{0}, Z_{1}$.

It is worthy to point out that the Hill-type formula (1.10) is also true for the complex Lagrangian boundary conditions, and the proof is similar as Theorem 1.1 in [13].

The trace formula can be derived from the Hill-type formula. Set

$$
\begin{aligned}
& P=\gamma_{0}^{-1}(T) Y-X, \\
& \hat{D}(t)=\gamma_{0}^{T}(t) D(t) \gamma_{0}(t), \\
& M_{j}=\int_{0}^{T} J \hat{D}\left(t_{1}\right) \int_{0}^{t_{1}} J \hat{D}\left(t_{2}\right) \cdots \int_{0}^{t_{j-1}} J \hat{D}\left(t_{j}\right) d t_{j} \cdots d t_{2} d t_{1} .
\end{aligned}
$$

Let $G_{j}=P^{-1} M_{j} X$ and $\mathcal{F}=\mathcal{F}(B, D)$ for simplicity. Then we get the following Krein-type trace formula.

Theorem 1.2. Under the above notations, we have for $m \in \mathbb{N}$,

$$
\operatorname{Tr}\left(\mathcal{F}^{m}\right)=m \sum_{k=1}^{m} \frac{1}{k}\left(\sum_{j_{1}+\cdots+j_{k}=m} \operatorname{Tr}\left(G_{j_{1}} \cdots G_{j_{k}}\right)\right) .
$$

In particular,

$$
\operatorname{Tr}(\mathcal{F})=\operatorname{Tr}\left(G_{1}\right), \quad \operatorname{Tr}\left(\mathcal{F}^{2}\right)=\operatorname{Tr}\left(G_{1}^{2}\right)+2 \operatorname{Tr}\left(G_{2}\right) .
$$

Since $\mathcal{F}^{m}$ is a trace class operator for $m \geq 2$, we have

$$
\operatorname{Tr}\left(\mathcal{F}^{m}\right)=\sum_{j} \frac{1}{\lambda_{j}^{m}}=m \sum_{k=1}^{m} \frac{1}{k}\left(\sum_{j_{1}+\cdots+j_{k}=m} \operatorname{Tr}\left(G_{j_{1}} \cdots G_{j_{k}}\right)\right),
$$

where the algebraic multiplicity of $\lambda_{j}$ is counted.

The original work of the trace formula is due to Krein $[19,20]$ in 1950's. In fact, Krein considered the following system

$$
\dot{z}(t)=\lambda J D(t) z(t), \quad z(0)=-z(T),
$$


where $D \geq 0$ and $\int_{0}^{T} D(t) d t>0$. System (1.13) is a special case of Hamiltonian system (1.3)-(1.4). For the system (1.13), Krein proved that

$$
\begin{aligned}
& \lim _{r \rightarrow \infty} \sum_{\left|\lambda_{j}\right|<r} \frac{1}{\lambda_{j}}=0, \\
& \sum \frac{1}{\lambda_{j}^{2}}=\frac{T^{2}}{2} \operatorname{Tr}\left(A_{11} A_{22}-A_{12}^{2}\right),
\end{aligned}
$$

where $\left\{\lambda_{j}\right\}$ are the eigenvalues for the system (1.13), and

$$
\left(\begin{array}{ll}
A_{11} & A_{12} \\
A_{21} & A_{22}
\end{array}\right)=\frac{1}{T} \int_{0}^{T} D(t) d t
$$

Moreover, Krein gave an interesting stability criterion:

$$
\frac{T^{2}}{2} \operatorname{Tr}\left(A_{11} A_{22}-A_{12}^{2}\right)<1 .
$$

Remark 1.3. Krein considered the simplest Hamiltonian system with some special conditions such as $D \geq 0$ and $\int_{0}^{T} D(t) d t>0$. For the system coming from $n$-body problem, these conditions are not satisfied. Motivated by Krein's idea, $\mathrm{Hu}, \mathrm{Ou}$ and Wang applied the trace formula for general boundary conditions to give nontrivial estimation of the stability region of elliptic Lagrangian orbits in planar three-body problem. The readers are referred to $[12,13]$ for details.

Next, we consider the Sturm-Liouville system

$$
-(P(t) \dot{y}(t)+Q(t) y(t))^{\cdot}+Q(t)^{T} \dot{y}(t)+\left(R(t)+\lambda R_{1}(t)\right) y(t)=0,
$$

where $P, Q \in W^{1,2}([0, T] ; \mathcal{M}(n)), R, R_{1} \in C([0, T] ; \mathcal{M}(n)), P(t)$ is invertible and $P(t)$, $R(t), R_{1}(t) \in \mathcal{S}(n)$ for $t \in[0, T]$. Let

$$
x(t)=P(t) \dot{y}(t)+Q(t) y(t) \quad \text { and } \quad z(t)=\left[\begin{array}{l}
x(t) \\
y(t)
\end{array}\right] .
$$

Then as in (1.4), any self-adjoint boundary condition can be written as

$$
\left[\begin{array}{l}
z(0) \\
z(T)
\end{array}\right] \in \Lambda,
$$

where

$$
\Lambda=\left[\begin{array}{l}
X \\
Y
\end{array}\right] \in \operatorname{Lag}(\mathcal{V}, \Omega) .
$$


By the Legendre transformation, (1.16) corresponds to the linear Hamiltonian system

$$
\dot{z}(t)=J_{n}(B(t)+\lambda D(t)) z(t),
$$

where

$$
B(t)=\left[\begin{array}{cc}
P(t)^{-1} & -P(t)^{-1} Q(t) \\
-Q(t)^{T} P(t)^{-1} & Q(t)^{T} P(t)^{-1} Q(t)-R(t)
\end{array}\right], \quad D(t)=\left[\begin{array}{cc}
0 & 0 \\
0 & -R_{1}(t)
\end{array}\right] .
$$

We define the operator

$$
\mathcal{A}=-\frac{d}{d t}\left(P \frac{d}{d t}+Q\right)+Q^{T} \frac{d}{d t}+R
$$

on $L^{2}\left([0, T] ; \mathbb{C}^{n}\right)$ with domain

$$
\mathbf{E}_{\Lambda}=\left\{y \in W^{2,2}\left([0, T] ; \mathbb{C}^{n}\right) \mid\left[\begin{array}{l}
z(0) \\
z(T)
\end{array}\right] \in \Lambda\right\}
$$

It is well-known that $\mathcal{A}$ is a self-adjoint operator. We assume that $\mathcal{A}$ is non-degenerate. Then $R_{1} \mathcal{A}^{-1}$ is a trace class operator and the classical Fredholm determinant $\operatorname{det}(I+$ $\lambda R_{1} \mathcal{A}^{-1}$ ) can be defined. We still use $\gamma_{\lambda}(t)$ to denote the fundamental solutions of (1.18) with the initial data $\gamma_{\lambda}(0)=I_{2 n}$. Now, we give the Hill-type formula for Sturm-Liouville system (1.16)-(1.17).

Theorem 1.3. Assume that $\mathcal{A}$ is non-degenerate, then

$$
\prod_{j}\left(1-\lambda_{j}^{-1}\right)=\operatorname{det}\left(\gamma_{1}(T) X-Y\right) \cdot \operatorname{det}\left(\gamma_{0}(T) X-Y\right)^{-1}
$$

where $\left\{-\lambda_{j}^{-1}\right\}$ are all the eigenvalues of $R_{1} \mathcal{A}^{-1}$ counting algebraic multiplicity, and $\left(X^{T}, Y^{T}\right)^{T}$ is a frame of $\Lambda$ in (1.17).

Similarily, we have the trace formula.

Theorem 1.4. Assume that $\mathcal{A}$ is non-degenerate, then for $m \in \mathbb{N}$,

$$
\sum_{j} \lambda_{j}^{-m}=m \sum_{k=1}^{m} \frac{1}{k}\left(\sum_{j_{1}+\cdots+j_{k}=m} \operatorname{Tr}\left(G_{j_{1}} \cdots G_{j_{k}}\right)\right)
$$

where $\left\{-\lambda_{j}^{-1}\right\}$ are all the eigenvalues of $R_{1} \mathcal{A}^{-1}$ counting algebraic multiplicity, and $G_{j}$ is the same as Theorem 1.2.

Finally, we give some applications of the trace formula. As an application, we will give some estimations on the non-degeneracy of the linear system. It is well-known that the system preserves the non-degeneracy under small perturbations. A natural question 
will arise: can we give an upper bound for the perturbation such that, under the smaller perturbation, the systems preserve the non-degeneracy? By the trace formula, we can answer this question partly. Details can be found in Section 5. As another application, the trace formula could be used to estimate the relative Morse index for Hamiltonian systems and the Morse index for Lagrangian systems. It is well-known that the relative Morse index (or Morse index) is equal to the Maslov-type index for the path of symplectic matrices and the Maslov-type index is a successful tool in judging the linear stability [14, 22]. We will not discuss the stability in the present paper, and interested readers could find the details in $[12,13]$.

We would like to point out that there are some other interesting applications of the Hill-type formula given by Portaluri and $\mathrm{Wu}$ [25], which relates to the spectral flow and degree theory.

The paper is organized as follows. Section 2 is devoted to preliminaries on conditional Fredholm determinant and conditional trace. In Section 3, the Hill-type formula and the Krein-type trace formula are proved for Hamiltonian systems with any selfadjoint boundary conditions. These two type's formula are proved for Sturm-Liouville systems with any self-adjoint boundary conditions in Section 4. Some estimations of relative Morse index and stability criteria are given in Section 5.

\section{Conditional Fredholm determinant and conditional trace}

In this section, we introduce some preliminary results, which include properties of the conditional Fredholm determinant and conditional trace developed in $[13,15]$.

The original idea of the conditional Fredholm determinant comes from [15]. For the classical theory of Fredholm determinant, if $C$ is a trace class operator on a Hilbert space $H$, then the Fredholm determinant $\operatorname{det}(I+\alpha C)$ is well-defined and it is an entire function on $\alpha$. In the study of Hamiltonian system, however, $A^{-1}$ is not necessarily a trace class operator but a Hilbert-Schmidt operator, where $A$ is assumed to be invertible. This makes the traditional Fredholm determinant not well-defined here. To break this barrier, the conditional Fredholm determinant was introduced in [15] for a class of Hilbert-Schmidt operator satisfying the following condition.

Definition 2.1. Let $\mathcal{P}=\left\{P_{k}\right\}$ be a sequence of orthogonal projections on the Hilbert space $H$ such that

1) Range $\left(P_{k}\right) \subset \operatorname{Range}\left(P_{m}\right)$ for $k \leq m$,

2) $P_{k}$ converges to I in strong operator topology.

A Hilbert-Schmidt operator $F$ is called to have the "trace finite condition" associated to $\mathcal{P}$ if the limit $\lim _{k \rightarrow \infty} \operatorname{Tr}\left(P_{k} F P_{k}\right)$ exists and is finite. In this case, we still use the notation $\operatorname{Tr}(F):=$ $\lim _{k \rightarrow \infty} \operatorname{Tr}\left(P_{k} F P_{k}\right)$, which is called the "conditional trace" of $F$ associated to $\mathcal{P}$. 
Denote by $\mathcal{J}(\mathcal{P})$ the set of Hilbert-Schmidt operators with trace finite condition associated to $\mathcal{P}$. It is easy to see that $\mathcal{J}(\mathcal{P})$ is a linear space and

$$
\mathcal{J}_{1} \subset \mathcal{J}(\mathcal{P}) \subset \mathcal{J}_{2}
$$

where $\mathcal{J}_{1}$ and $\mathcal{J}_{2}$ are the ideals of trace class operators and Hilbert-Schmidt operators, respectively. In general, $\mathcal{J}(\mathcal{P})$ is not an ideal of the algebra of bounded linear operators.

For $F \in \mathcal{J}_{2}$, the regularized Fredholm determinant can be defined (see, for example, [27]):

$$
\operatorname{det}_{2}(I+F)=\operatorname{det}\left((I+F) e^{-F}\right) .
$$

Denote by $\|F\|_{2}$ the Hilbert-Schmidt norm for $F \in \mathcal{J}_{2}$. Then for any sequence of finite rank operators $\left\{F_{k}\right\}$ such that $\left\|F_{k}-F\right\|_{2} \rightarrow 0$ as $k \rightarrow \infty$, we have

$$
\operatorname{det}_{2}(I+F)=\lim _{k \rightarrow \infty} \operatorname{det}_{2}\left(I+F_{k}\right) .
$$

Therefore, if $F \in \mathcal{J}_{2}$ has the trace finite condition associated to $\left\{P_{k}\right\}$, then setting $F_{k}=$ $P_{k} F P_{k}$, we have

$$
\begin{aligned}
\operatorname{det}_{2}(I+F) & =\lim _{k \rightarrow \infty} \operatorname{det}_{2}\left(I+F_{k}\right)=\lim _{k \rightarrow \infty} \operatorname{det}\left(\left(I+F_{k}\right) e^{-F_{k}}\right) \\
& =\lim _{k \rightarrow \infty} \operatorname{det}\left(I+F_{k}\right) e^{-\operatorname{Tr}\left(F_{k}\right)}=\lim _{k \rightarrow \infty} \operatorname{det}\left(I+F_{k}\right) e^{-\lim _{k \rightarrow \infty} \operatorname{Tr}\left(F_{k}\right)},
\end{aligned}
$$

which means

$$
\lim _{k \rightarrow \infty} \operatorname{det}\left(I+F_{k}\right)=\operatorname{det}_{2}(I+F) e^{\lim _{k \rightarrow \infty} \operatorname{Tr}\left(F_{k}\right)}
$$

is convergent. To simplify the notation, we have the following definition.

Definition 2.2. If $F \in \mathcal{J}_{2}$ has the trace finite condition associated to $\left\{P_{k}\right\}$, then we define the conditional Fredholm determinant

$$
\operatorname{det}(I+F)=\lim _{k \rightarrow \infty} \operatorname{det}\left(I+F_{k}\right)
$$

where $F_{k}=P_{k} F P_{k}$.

The conditional Fredholm determinant shares many properties with the classical Fredholm determinant. For example, in $[12,15]$, by using Montel's Theorem, we have

Lemma 2.1. If $F \in \mathcal{J}_{2}$ has the trace finite condition, then the function $\operatorname{det}(I+\alpha F)$ is an entire function on the variable $\alpha$.

The next lemma collects some basic properties of the determinant.

Lemma 2.2 (Theorem 3.5 in [15]). If $D, F \in \mathcal{J}_{2}$ have the trace finite condition, then 
1) $\overline{\operatorname{det}(I+D)}=\operatorname{det}\left(I+D^{*}\right)$,

2) $\operatorname{det}(I+D) \operatorname{det}(I+F)=\operatorname{det}(I+D+F+D F)$,

3) $\operatorname{det}(I+D) \neq 0$ if and only if $I+D$ is invertible,

4) for any $\lambda_{0} \neq 0$, set $z_{0}=-\lambda_{0}^{-1}$, then $\lambda_{0}$ is an eigenvalue of $D$ of algebraic multiplicity $k$ if and only if $\lambda_{0}$ is a zero point of $\operatorname{det}(I+\alpha D)$ of order $k$.

Suppose $V_{0}, V_{1} \in \operatorname{Lag}\left(\mathbb{C}^{2 n}, \omega_{n}\right)$. When we consider the Lagrangian boundary conditions, the domain of $\left.A\right|_{E_{V_{0}, V_{1}}}$ is defined as

$$
E_{V_{0}, V_{1}}=\left\{z(t) \in W^{1,2}\left([0, T] ; \mathbb{C}^{2 n}\right) \mid z(0) \in V_{0}, z(T) \in V_{1}\right\} .
$$

Following [13], we may change a symplectic basis and assume that the Lagrangian frames of $V_{0}, V_{1}$ are

$$
Z_{0}=\left(I_{n}, 0_{n}\right)^{T}, \quad Z_{1}=(C(\theta), S(\theta))^{T},
$$

respectively, where for $-\pi / 2<\theta_{j} \leq \pi / 2$,

$$
C(\theta)=\operatorname{diag}\left(\cos \left(\theta_{1}\right), \cdots, \cos \left(\theta_{n}\right)\right), \quad S(\theta)=\operatorname{diag}\left(\sin \left(\theta_{1}\right), \cdots, \sin \left(\theta_{n}\right)\right) .
$$

Then

$$
\sigma_{p}\left(\left.A\right|_{E_{V_{0}, V_{1}}}\right)=\left\{\lambda_{j, k} \mid \lambda_{j, k}=\theta_{j} / T+k \pi / T, j=1,2, \cdots, n, k \in \mathbb{Z}\right\},
$$

with the corresponding eigenfunctions

$$
e_{j, k}=e^{\lambda_{j, k} t t} e_{j}=\cos \left(\lambda_{j, k} t\right) e_{j}+\sin \left(\lambda_{j, k} t\right) e_{n+j},
$$

where $e_{j}$ is the standard $j$-th basis of $\mathbb{C}^{2 n}$. In the following lemma. Let $P_{N}$ be the projections from $\mathcal{H}$ to $\operatorname{span}\left\{e_{j, k}: 1 \leq j \leq n,|k| \leq N\right\}$.

Lemma 2.3 (Proposition 2.2 in [13]).

i) For any $v \in \mathbb{C}$ such that $\left.A\right|_{E_{V_{0}, V_{1}}}-v$ is invertible, $\mathcal{F}\left(v, D ; E_{V_{0}, V_{1}}\right) \in \mathcal{J}\left(\left\{\mathcal{P}_{\mathcal{N}}\right\}\right)$ and

$$
\begin{aligned}
\operatorname{Tr} \mathcal{F}\left(v, D ; E_{V_{0}, V_{1}}\right)=\sum_{j=1}^{n} & \frac{\cos \left(\theta_{j}-T v\right)}{\sin \left(\theta_{j}-T v\right)} \int_{0}^{T}\left(e^{-v J t} D e^{v J t} e_{j}, e_{j}\right) d t \\
& +\sum_{j=1}^{n} \int_{0}^{T}\left(e^{-v J t} D e^{v J t} e_{n+j}, e_{j}\right) d t
\end{aligned}
$$

ii) $\mathcal{F}\left(B, D ; E_{V_{0}, V_{1}}\right) \in \mathcal{J}\left(\left\{\mathcal{P}_{\mathcal{N}}\right\}\right)$ and hence the conditional Fredholm determinant $\operatorname{det}(I-$ $\left.\mathcal{F}\left(B, D ; E_{V_{0}, V_{1}}\right)\right)$ is well defined. 


\section{Hill-type formula and Krein-type trace formula for Hamiltonian systems}

In this section, we prove the Hill-type formula for Hamiltonian systems with any selfadjoint boundary condition. By Taylor expansion of the parameterized Hill-type formula, we derive the Krein-type trace formula.

\subsection{Hill-type formula for Hamiltonian systems with any self-adjoint boundary condition}

Recall that the Hamiltonian system with any self-adjoint boundary condition takes the form (1.3)-(1.4), and the frame $\left[\begin{array}{l}X \\ Y\end{array}\right]$ of the boundary condition $\Lambda$ in (1.4) satisfies (1.5). Let

$$
X=\left[\begin{array}{ll}
X_{11} & X_{12} \\
X_{21} & X_{22}
\end{array}\right] \text { and } Y=\left[\begin{array}{ll}
Y_{11} & Y_{12} \\
Y_{21} & Y_{22}
\end{array}\right]
$$

where $X_{i j}, Y_{i j} \in \mathcal{M}(n)$. Then by (1.5) we have

$$
\left[\begin{array}{ll}
X_{21}^{*} X_{11}-X_{11}^{*} X_{21} & X_{21}^{*} X_{12}-X_{11}^{*} X_{22} \\
X_{22}^{*} X_{11}-X_{12}^{*} X_{21} & X_{22}^{*} X_{12}-X_{12}^{*} X_{22}
\end{array}\right]=\left[\begin{array}{ll}
Y_{21}^{*} Y_{11}-Y_{11}^{*} Y_{21} & Y_{21}^{*} Y_{12}-Y_{11}^{*} Y_{22} \\
Y_{22}^{*} Y_{11}-Y_{12}^{*} Y_{21} & Y_{22}^{*} Y_{12}-Y_{12}^{*} Y_{22}
\end{array}\right]
$$

Let

$$
\begin{aligned}
z(t)=\left[\begin{array}{l}
z_{1}(t) \\
z_{2}(t)
\end{array}\right], & \tilde{z}(t)=\left[\begin{array}{l}
\tilde{z}_{1}(t) \\
\tilde{z}_{2}(t)
\end{array}\right], \\
B(t)=\left[\begin{array}{ll}
b_{11}(t) & b_{12}(t) \\
b_{12}(t)^{T} & b_{22}(t)
\end{array}\right], & D(t)=\left[\begin{array}{cc}
d_{11}(t) & d_{12}(t) \\
d_{12}(t)^{T} & d_{22}(t)
\end{array}\right],
\end{aligned}
$$

where $z_{1}(t), z_{2}(t), \tilde{z}_{1}(t), \tilde{z}_{2}(t) \in \mathbb{C}^{n}, b_{i i}(t), d_{i i}(t) \in \mathcal{S}(n)$, and $b_{i j}(t), d_{i j}(t) \in \mathcal{M}(n)$ for $i \neq$ $j$. Now, we construct a $4 n$-dimensional Hamiltonian system, which satisfies a complex Lagrangian boundary condition and thus allows us to apply (1.10) in Remark 1.2 later. Consider a $4 n$-dimensional Hamiltonian system

$$
\left[\begin{array}{l}
\dot{\tilde{z}}_{2}(t) \\
\dot{z}_{1}(t) \\
\dot{\tilde{z}}_{1}(t) \\
\dot{z}_{2}(t)
\end{array}\right]=J_{2 n}\left(\left[\begin{array}{cccc}
0 & 0 & 0 & 0 \\
0 & b_{11}(t) & 0 & b_{12}(t) \\
0 & 0 & 0 & 0 \\
0 & b_{12}(t)^{T} & 0 & b_{22}(t)
\end{array}\right]+\lambda\left[\begin{array}{cccc}
0 & 0 & 0 & 0 \\
0 & d_{11}(t) & 0 & d_{12}(t) \\
0 & 0 & 0 & 0 \\
0 & d_{12}(t)^{T} & 0 & d_{22}(t)
\end{array}\right]\right)\left[\begin{array}{c}
\tilde{z}_{2}(t) \\
z_{1}(t) \\
\tilde{z}_{1}(t) \\
z_{2}(t)
\end{array}\right]
$$

with the boundary condition

$$
\left[\begin{array}{l}
\tilde{z}_{2}(0) \\
z_{1}(0) \\
\tilde{z}_{1}(0) \\
z_{2}(0)
\end{array}\right] \in\left[\begin{array}{cc}
0 & I_{n} \\
I_{n} & 0 \\
I_{n} & 0 \\
0 & I_{n}
\end{array}\right]=: \Lambda_{0} \text { and }\left[\begin{array}{c}
\tilde{z}_{2}(T) \\
z_{1}(T) \\
\tilde{z}_{1}(T) \\
z_{2}(T)
\end{array}\right] \in\left[\begin{array}{cc}
X_{21} & X_{22} \\
Y_{11} & Y_{12} \\
X_{11} & X_{12} \\
Y_{21} & Y_{22}
\end{array}\right]=: \Lambda_{T}
$$


where we identify the subspaces $\Lambda_{0}$ and $\Lambda_{T}$ with their frames. To confirm that (3.3) is a complex Lagrangian boundary condition, we need to check that $\Lambda_{0}$ and $\Lambda_{T}$ are Lagrangian subspaces in $\left(\mathbb{C}^{4 n}, \omega_{2 n}\right)$. Note that the basis here is $\omega_{2 n}$, and thus we will verify that

$$
X_{i}^{*} Y_{i}=Y_{i}^{*} X_{i} \quad \text { for } \quad \Lambda_{i}=\left[\begin{array}{c}
X_{i} \\
Y_{i}
\end{array}\right], \quad i=0, T .
$$

It is evident that $\Lambda_{0}$ is Lagrangian. Direct computation implies

$$
\begin{aligned}
& {\left[\begin{array}{ll}
X_{21}^{*} & Y_{11}^{*} \\
X_{22}^{*} & Y_{12}^{*}
\end{array}\right]\left[\begin{array}{ll}
X_{11} & X_{12} \\
Y_{21} & Y_{22}
\end{array}\right]=\left[\begin{array}{ll}
X_{21}^{*} X_{11}+Y_{11}^{*} Y_{21} & X_{21}^{*} X_{12}+Y_{11}^{*} Y_{22} \\
X_{22}^{*} X_{11}+Y_{12}^{*} Y_{21} & X_{22}^{*} X_{12}+Y_{12}^{*} Y_{22}
\end{array}\right],} \\
& {\left[\begin{array}{ll}
X_{11}^{*} & Y_{21}^{*} \\
X_{12}^{*} & Y_{22}^{*}
\end{array}\right]\left[\begin{array}{ll}
X_{21} & X_{22} \\
Y_{11} & Y_{12}
\end{array}\right]=\left[\begin{array}{ll}
X_{11}^{*} X_{21}+Y_{21}^{*} Y_{11} & X_{11}^{*} X_{22}+Y_{21}^{*} Y_{12} \\
X_{12}^{*} X_{21}+Y_{22}^{*} Y_{11} & X_{12}^{*} X_{22}+Y_{22}^{*} Y_{12}
\end{array}\right] .}
\end{aligned}
$$

Then by (3.1), we have

$$
\left[\begin{array}{ll}
X_{21}^{*} & Y_{11}^{*} \\
X_{22}^{*} & Y_{12}^{*}
\end{array}\right]\left[\begin{array}{ll}
X_{11} & X_{12} \\
Y_{21} & Y_{22}
\end{array}\right]=\left[\begin{array}{ll}
X_{11}^{*} & Y_{21}^{*} \\
X_{12}^{*} & Y_{22}^{*}
\end{array}\right]\left[\begin{array}{cc}
X_{21} & X_{22} \\
Y_{11} & Y_{12}
\end{array}\right]
$$

which verifies that (3.3) is a complex Lagrangian boundary condition. Let

$$
\hat{z}(t)=\left[\begin{array}{l}
\tilde{z}_{2}(t) \\
z_{1}(t) \\
\tilde{z}_{1}(t) \\
z_{2}(t)
\end{array}\right]
$$

and define the operator

$$
\hat{A}=-J_{2 n} \frac{d}{d t} \quad \text { on } \quad L^{2}\left([0, T] ; \mathbb{C}^{4 n}\right)
$$

with domain

$$
E_{\Lambda_{0}, \Lambda_{T}}=\left\{\hat{z} \in W^{1,2}\left([0, T] ; \mathbb{C}^{4 n}\right) \mid \hat{z}(0) \in \Lambda_{0}, \hat{z}(T) \in \Lambda_{T}\right\} .
$$

Then $\hat{A}$ is a self-adjoint operator and has compact resolvent. Then by the discussion in (2.2), we have

$$
\sigma(\hat{A})=\left\{\hat{\lambda}_{j, k} \mid \hat{\lambda}_{j, k}=\theta_{j} / T+k \pi / T, 1 \leq j \leq 2 n, k \in \mathbb{Z}\right\} .
$$

The corresponding eigenfunctions are denoted by

$$
\hat{e}_{j, k}=\left(\hat{e}_{j, k, l}\right)_{1 \leq l \leq 4}=\left[\begin{array}{c}
\hat{e}_{j, k, 1} \\
\hat{e}_{j, k, 2} \\
\hat{e}_{j, k, 3} \\
\hat{e}_{j, k, 4}
\end{array}\right],
$$


where $\hat{e}_{j, k, l} \in L^{2}\left([0, T] ; \mathbb{C}^{n}\right), 1 \leq l \leq 4$. Let $\hat{P}_{N}$ be the projections from $L^{2}\left([0, T] ; \mathbb{C}^{4 n}\right)$ to span $\left\{\hat{e}_{j, k}: 1 \leq j \leq 2 n,|k| \leq N\right\}$, and $P_{N}$ be the projections from $L^{2}\left([0, T] ; \mathbb{C}^{2 n}\right)$ to

$$
\operatorname{span}\left\{\left[\begin{array}{l}
\hat{e}_{j, k, 2} \\
\hat{e}_{j, k, 4}
\end{array}\right]: 1 \leq j \leq 2 n,|k| \leq N\right\} \text {, }
$$

respectively. Then Range $\left(P_{N}\right) \subset \operatorname{Range}\left(P_{M}\right)$ for $N \leq M$ and $P_{N}$ converges to the identity $I$ on $L^{2}\left([0, T] ; \mathbb{C}^{2 n}\right)$ in the strong operator topology. $\hat{P}_{N}$ has similar properties.

Recall that

$$
\tilde{z}(t)=\left[\begin{array}{c}
\tilde{z}_{1}(t) \\
\tilde{z}_{2}(t)
\end{array}\right] \quad \text { and } \quad z(t)=\left[\begin{array}{l}
z_{1}(t) \\
z_{2}(t)
\end{array}\right]
$$

Then the Hamiltonian system (3.2)-(3.3) can be rewritten as

$$
\left\{\begin{array}{l}
\dot{z}(t)=0 \\
\dot{z}(t)=J_{n}(B(t)+\lambda D(t)) z(t),
\end{array}\right.
$$

with the boundary condition

$$
\left[\begin{array}{l}
\tilde{z}(0) \\
z(0)
\end{array}\right] \in\left[\begin{array}{l}
I_{2 n} \\
I_{2 n}
\end{array}\right] \text { and }\left[\begin{array}{l}
\tilde{z}(T) \\
z(T)
\end{array}\right] \in\left[\begin{array}{l}
X \\
Y
\end{array}\right]=\Lambda
$$

Moreover, we have the following conclusion.

Lemma 3.1. $z(t)$ is a solution of (1.3)-(1.4) if and only if $\left[\begin{array}{l}z(0) \\ z(t)\end{array}\right]$ is a solution of (3.4)-(3.5).

Following [22], denote

$$
M \diamond N=\left[\begin{array}{cccc}
m_{11} & 0 & m_{12} & 0 \\
0 & n_{11} & 0 & n_{12} \\
m_{21} & 0 & m_{22} & 0 \\
0 & n_{21} & 0 & n_{22}
\end{array}\right]
$$

for

$$
M=\left[\begin{array}{ll}
m_{11} & m_{12} \\
m_{21} & m_{22}
\end{array}\right], \quad N=\left[\begin{array}{ll}
n_{11} & n_{12} \\
n_{21} & n_{22}
\end{array}\right] \in \mathcal{M}(2 n),
$$

and $m_{i j}, n_{i j} \in \mathcal{M}(n)$. Let

$$
\hat{B}(t)=0_{2 n} \diamond B(t) \quad \text { and } \quad \hat{D}(t)=0_{2 n} \diamond D(t) .
$$

Then by Lemma 2.3 ii), we have

$$
\operatorname{Tr}\left(\hat{D}(\hat{A}-\hat{B})^{-1}\right)=\lim _{N \rightarrow \infty} \operatorname{Tr}\left(\hat{P}_{N}\left(\hat{D}(\hat{A}-\hat{B})^{-1}\right) \hat{P}_{N}\right)
$$


exists and is finite. Thus, $\operatorname{det}\left(I-\hat{D}(\hat{A}-\hat{B})^{-1}\right)$ is well-defined. By the special choice of $P_{N}$, we have

$$
\operatorname{Tr}\left(\hat{P}_{N}\left(\hat{D}(\hat{A}-\hat{B})^{-1}\right) \hat{P}_{N}\right)=\operatorname{Tr}\left(P_{N}\left(D(A-B)^{-1}\right) P_{N}\right),
$$

and thus,

$$
\operatorname{Tr}\left(D(A-B)^{-1}\right)=\lim _{N \rightarrow \infty} \operatorname{Tr}\left(P_{N}\left(D(A-B)^{-1}\right) P_{N}\right)
$$

exists and is finite. Hence, the conditional Fredholm determinant $\operatorname{det}\left(I-D(A-B)^{-1}\right)$ is well defined associated to the projections $\left\{P_{N}\right\}$. Denote

$$
\gamma_{\lambda}(t)=\left[\begin{array}{ll}
\gamma_{\lambda, 11}(t) & \gamma_{\lambda, 12}(t) \\
\gamma_{\lambda, 21}(t) & \gamma_{\lambda, 22}(t)
\end{array}\right]
$$

the fundamental solutions of (1.3) with the initial data $\gamma_{\lambda}(0)=I_{2 n}$. Then $I_{2 n} \diamond \gamma_{\lambda}(t)$ is the fundamental solutions of (3.2) with the initial data $I_{2 n} \diamond \gamma_{\lambda}(0)=I_{4 n}$. Now, we are in a position to prove Theorem 1.1: the Hill-type formula for Hamiltonian system (1.3)-(1.4).

Proof of Theorem 1.1. Since $A-B$ is non-degenerate, we have $z(t) \equiv 0$ is the only solution of (1.3)-(1.4) for $\lambda=0$. By Lemma 3.1,

$$
\left[\begin{array}{l}
\tilde{z}(t) \\
z(t)
\end{array}\right] \equiv\left[\begin{array}{l}
0 \\
0
\end{array}\right]
$$

is the only solution of (3.4)-(3.5) for $\lambda=0$. Thus, $\hat{A}-\hat{B}$ is non-degenerate. Since (3.3) is a Lagrangian boundary condition, we apply (1.10) in Remark 1.2 to (3.2)-(3.3) to get

$$
\begin{aligned}
& \operatorname{det}\left(I-\hat{D}(\hat{A}-\hat{B})^{-1}\right)=\lim _{N \rightarrow \infty} \operatorname{det}\left(I-\hat{P}_{N} \hat{D}(\hat{A}-\hat{B})^{-1} \hat{P}_{N}\right) \\
= & \operatorname{det}\left(\left(I_{2 n} \diamond \gamma_{1}(T)\right) \Lambda_{0}, \Lambda_{T}\right) \cdot \operatorname{det}\left(\left(I_{2 n} \diamond \gamma_{0}(T)\right) \Lambda_{0}, \Lambda_{T}\right)^{-1} .
\end{aligned}
$$

For $k=0,1$, we have

$$
\begin{aligned}
& \operatorname{det}\left(\left(I_{2 n} \diamond \gamma_{k}(T)\right) \Lambda_{0}, \Lambda_{T}\right)=\operatorname{det}\left(\left[\begin{array}{cccc}
I_{n} & 0 & 0 & 0 \\
0 & \gamma_{k, 11}(T) & 0 & \gamma_{k, 12}(T) \\
0 & 0 & I_{n} & 0 \\
0 & \gamma_{k, 21}(T) & 0 & \gamma_{k, 22}(T)
\end{array}\right]\left[\begin{array}{cc}
0 & I_{n} \\
I_{n} & 0 \\
I_{n} & 0 \\
0 & I_{n}
\end{array}\right],\left[\begin{array}{cc}
X_{21} & X_{22} \\
Y_{11} & Y_{12} \\
X_{11} & X_{12} \\
Y_{21} & Y_{22}
\end{array}\right]\right) \\
& =\operatorname{det}\left(\left[\begin{array}{cccc}
0 & I_{n} & X_{21} & X_{22} \\
\gamma_{k, 11}(T) & \gamma_{k, 12}(T) & Y_{11} & Y_{12} \\
I_{n} & 0 & X_{11} & X_{12} \\
\gamma_{k, 21}(T) & \gamma_{k, 22}(T) & Y_{21} & Y_{22}
\end{array}\right]\right)=(-1)^{2 n} \operatorname{det}\left(\left[\begin{array}{cccc}
I_{n} & 0 & X_{11} & X_{12} \\
0 & I_{n} & X_{21} & X_{22} \\
\gamma_{k, 11}(T) & \gamma_{k, 12}(T) & Y_{11} & X_{12} \\
\gamma_{k, 21}(T) & \gamma_{k, 22}(T) & Y_{21} & Y_{22}
\end{array}\right]\right) \\
& =\operatorname{det}\left(\left[\begin{array}{cc}
I_{2 n} & X \\
\gamma_{k}(T) & Y
\end{array}\right]\right)=\operatorname{det}\left(Y-\gamma_{k}(T) X\right) \text {. }
\end{aligned}
$$

Then we prove that

$$
\operatorname{det}_{2}\left(I-\hat{D}(\hat{A}-\hat{B})^{-1}\right)=\operatorname{det}_{2}\left(I-D(A-B)^{-1}\right) .
$$


Assume that (3.9) is true. By (3.6) we have

$$
\begin{aligned}
& \operatorname{det}\left(I-\hat{D}(\hat{A}-\hat{B})^{-1}\right)=\operatorname{det}_{2}\left(I-\hat{D}(\hat{A}-\hat{B})^{-1}\right) \lim _{N \rightarrow \infty} e^{\operatorname{Tr}\left(-\hat{P}_{N} \hat{D}(\hat{A}-\hat{B})^{-1} \hat{P}_{N}\right)} \\
= & \operatorname{det}_{2}\left(I-D(A-B)^{-1}\right) \lim _{N \rightarrow \infty} e^{\operatorname{Tr}\left(-P_{N} D(A-B)^{-1} P_{N}\right)}=\operatorname{det}\left(I-D(A-B)^{-1}\right) .
\end{aligned}
$$

Then (1.8) follows from (3.10), (3.8) and (3.7).

It rests to prove (3.9). By Lemma 3.1,

$$
\sigma\left(\hat{D}(\hat{A}-\hat{B})^{-1}\right)=\sigma\left(D(A-B)^{-1}\right) .
$$

We will prove that the algebraic multiplicity of $\lambda$ as an eigenvalue of $\hat{D}(\hat{A}-\hat{B})^{-1}$ is the same as that of $\lambda$ as an eigenvalue of $D(A-B)^{-1}$. Suppose that $\hat{v}_{1}$ is an eigenfunction of $\lambda \in \sigma\left(\hat{D}(\hat{A}-\hat{B})^{-1}\right)$. Let

$$
\hat{u}_{1}=\left(\hat{u}_{1, j}\right)_{1 \leq j \leq 4}:=(\hat{A}-\hat{B})^{-1} \hat{v}_{1},
$$

where $\hat{u}_{1, j} \in L^{2}\left([0, T] ; \mathbb{C}^{n}\right)$. Since

$$
(\hat{A}-\hat{B}) \hat{u}_{1}=\lambda^{-1} \hat{D} \hat{u}_{1}
$$

and $\hat{u}_{1}$ satisfies the boundary condition (3.3), we have

$$
\hat{u}_{1}(t)=\left(\hat{u}_{1,4}(0)^{T}, u_{1,2}(t)^{T}, \hat{u}_{1,2}(0)^{T}, \hat{u}_{1,4}(t)^{T}\right)^{T} \quad \text { and } \quad\left(\hat{u}_{1, j}\right)_{j=2,4} \in E_{\Lambda} .
$$

Let

$$
v_{1}=(A-B)\left(\hat{u}_{1, j}\right)_{j=2,4} .
$$

Then $v_{1}$ is an eigenfunction of $\lambda \in \sigma\left(D(A-B)^{-1}\right)$, and vice versa. Suppose that $\hat{v}_{2}$ is a generalized eigenfunction of $\lambda \in \sigma\left(\hat{D}(\hat{A}-\hat{B})^{-1}\right)$ satisfying

$$
\left(\hat{D}(\hat{A}-\hat{B})^{-1}-\lambda\right) \hat{v}_{2}=\hat{v}_{1} .
$$

Let

$$
\hat{u}_{2}=\left(\hat{u}_{2, j}\right)_{1 \leq j \leq 4}:=(\hat{A}-\hat{B})^{-1} \hat{v}_{2} .
$$

Then

$$
(\hat{A}-\hat{B})\left(\hat{u}_{2}+\lambda^{-1} \hat{u}_{1}\right)=\lambda^{-1} \hat{D} \hat{u}_{2}
$$

Denoting

$$
w=\left(w_{j}\right)_{1 \leq j \leq 4}:=\left(\hat{u}_{2, j}+\lambda^{-1} \hat{u}_{1, j}\right)_{1 \leq j \leq 4}
$$

we have

$$
w(t)=\left(w_{4}(0)^{T}, w_{2}(t)^{T}, w_{2}(0)^{T}, w_{4}(t)^{T}\right)^{T}
$$

and thus, $\left(w_{j}\right)_{j=2,4} \in E_{\Lambda}$. Then

$$
\begin{aligned}
& \left(\hat{u}_{2, j}\right)_{j=2,4}=\left(w_{j}-\lambda^{-1} \hat{u}_{1, j}\right)_{j=2,4} \in E_{\Lambda} \\
& (A-B)\left(\hat{u}_{2, j}+\lambda^{-1} \hat{u}_{1, j}\right)_{j=2,4}=\lambda^{-1} D\left(\hat{u}_{2, j}\right)_{j=2,4} .
\end{aligned}
$$


Let

$$
v_{2}=(A-B)\left(\hat{u}_{2, j}\right)_{j=2,4} .
$$

Then $v_{2}$ is a generalized eigenfunction of $\lambda \in \sigma\left(D(A-B)^{-1}\right)$ satisfying

$$
\left(D(A-B)^{-1}-\lambda\right) v_{2}=v_{1}
$$

and vice versa. If the algebraic multiplicity of $\lambda$ as an eigenvalue of $\hat{D}(\hat{A}-\hat{B})^{-1}$ is $k_{0}$ and

$$
\left(\hat{D}(\hat{A}-\hat{B})^{-1}-\lambda\right) \hat{v}_{i+1}=\hat{v}_{i} \quad \text { for } \quad 2 \leq i \leq k_{0}-1,
$$

by induction we can construct a corresponding generalized eigenfunction $v_{i+1}$ of $\lambda \in$ $\sigma\left(D(A-B)^{-1}\right)$ satisfying

$$
\left(D(A-B)^{-1}-\lambda\right) v_{i+1}=v_{i}
$$

and vice versa.

Denote $R(F)=(I+F) e^{-F}-I$ for an operator $F \in \mathcal{J}_{2}$ and $\left\{\lambda_{j}\right\}$ be all the eigenvalues of $\hat{D}(\hat{A}-\hat{B})^{-1}$, where the algebraic multiplicity is counted. Then $R\left(-\hat{D}(\hat{A}-\hat{B})^{-1}\right) \in \mathcal{J}_{1}$ and all the eigenvalues of $R\left(-\hat{D}(\hat{A}-\hat{B})^{-1}\right)$ are $\left\{\left(1-\lambda_{j}\right) e^{\lambda_{j}}-1\right\}$. Meanwhile, $R(-D(A-$ $\left.B)^{-1}\right) \in \mathcal{J}_{1}$ and all the eigenvalues of $R\left(-D(A-B)^{-1}\right)$ are $\left\{\left(1-\lambda_{j}\right) e^{\lambda_{j}}-1\right\}$. By Theorem 3.7 in [27], we have

$$
\begin{aligned}
\operatorname{det}_{2}\left(I-\hat{D}(\hat{A}-\hat{B})^{-1}\right) & =\operatorname{det}\left(I+R\left(-\hat{D}(\hat{A}-\hat{B})^{-1}\right)\right)=\prod_{j}\left(1+\left(1-\lambda_{j}\right) e^{\lambda_{j}}-1\right) \\
& =\operatorname{det}\left(I+R\left(-D(A-B)^{-1}\right)\right)=\operatorname{det}_{2}\left(I-D(A-B)^{-1}\right) .
\end{aligned}
$$

This proves (3.9).

\subsection{Krein-type trace formula for Hamiltonian systems with any self-adjoint boundary condition}

In this subsection, we derive the Krein-type trace formula for Hamiltonian systems with any self-adjoint boundary condition. The idea is similar as Section 2 in [12]. First, we study the Taylor expansion of the parameterized conditional Fredholm determinant and the linearly parameterized monodromy matrices, separately. Then we obtain the Kreintype trace formula by comparing the coefficients of the expansions in the parameterized Hill-type formula.

Recall that $\mathcal{F}=\mathcal{F}(B, D)$ and the conditional Fredholm determinant $\operatorname{det}(I-\alpha \mathcal{F})$ is well-defined associated to $\left\{P_{N}\right\}$, which is given in Subsection 3.1. Then by Theorem 2.6 in [16] we have

$$
\operatorname{det}(I-\alpha \mathcal{F})=\exp \left(-\sum_{m=1}^{\infty} \frac{1}{m} \operatorname{Tr}\left(\mathcal{F}^{m}\right) \alpha^{m}\right)
$$


Next, we consider the expansion of the parameterized monodromy matrices. Let

$$
M(\alpha)=Y-\gamma_{\alpha}(T) X, \quad f(\alpha)=\operatorname{det} M(\alpha) \operatorname{det} M(0)^{-1} \quad \text { and } \quad P=\gamma_{0}(T)^{-1} Y-X
$$

Then

$$
\begin{aligned}
f(\alpha) & =\operatorname{det}\left(\gamma_{0}(T)^{-1}\left(Y-\gamma_{\alpha}(T) X\right)\right) \operatorname{det}\left(P^{-1}\right) \\
& =\operatorname{det}\left(I_{2 n}-P^{-1}\left(\gamma_{0}(T)^{-1} \gamma_{\alpha}(T)-I_{2 n}\right) X\right) .
\end{aligned}
$$

Following Subsection 2.2 in [12], we have

$$
\gamma_{0}(T)^{-1} \gamma_{\alpha}(T)-I_{2 n}=\sum_{j=1}^{\infty} \alpha^{j} M_{j}
$$

where $M_{j}$ is defined in (1.11). Then

$$
f(\alpha)=\operatorname{det}\left(I_{2 n}-\sum_{j=1}^{\infty} \alpha^{j} G_{j}\right)
$$

where $G_{j}=P^{-1} M_{j} X$. Similar to (2.6) in [12], we have $f(\alpha)=e^{g(\alpha)}$, where

$$
\frac{g^{(m)}(0)}{m !}=\sum_{k=1}^{m} \frac{-1}{k}\left(\sum_{j_{1}+\cdots+j_{k}=m} \operatorname{Tr}\left(G_{j_{1}} \cdots G_{j_{k}}\right)\right) .
$$

Now, we give the proof of Theorem 1.2.

Proof of Theorem 1.2. By Theorem 1.1, we get the parameterized Hill-type formula det $(I-$ $\alpha \mathcal{F})=f(\alpha)$. Then the proof is done by comparing the coefficients of (3.11) and (3.12).

\section{Hill-type formula and Krein-type trace formula for the Sturm-Liouville system with any self-adjoint boundary condition}

In this section, we prove the Hill-type formula and the Krein-type trace formula for the Sturm-Liouville system (1.16)-(1.17). Recall that

$$
\mathcal{A}=-\frac{d}{d t}\left(P \frac{d}{d t}+Q\right)+Q^{T} \frac{d}{d t}+R
$$

is defined on $L^{2}\left([0, T] ; \mathbb{C}^{n}\right)$ with domain $\mathrm{E}_{\Lambda}$. If $\mathcal{A}$ is invertible, then $R_{1} \mathcal{A}^{-1} \in \mathcal{J}_{1}$. Thus, the classical Fredholm determinant $\operatorname{det}\left(I+\lambda R_{1} \mathcal{A}^{-1}\right)$ can be defined. We need the following lemma, where $\gamma_{\lambda}(t)$ is the fundamental solutions of (1.18) with the initial data $\gamma_{\lambda}(0)=I_{2 n}$, and $\left(X^{T}, Y^{T}\right)^{T}$ is a frame of $\Lambda$ in (1.17). 


\section{Lemma 4.1.}

i) $\operatorname{det}\left(I+\lambda_{0} R_{1} \mathcal{A}^{-1}\right)=0$ if and only if $\operatorname{det}\left(Y-\gamma_{\lambda_{0}}(T) X\right)=0$.

ii) Suppose $R_{1}>0$. Then the order of $\operatorname{det}\left(I+\lambda R_{1} \mathcal{A}^{-1}\right)$ at a zero point $\lambda_{0}$ is the same as that of $\operatorname{det}\left(Y-\gamma_{\lambda}(T) X\right)$ at $\lambda_{0}$.

Proof. i) $\operatorname{det}\left(I+\lambda_{0} R_{1} \mathcal{A}^{-1}\right)=0 \Longleftrightarrow-1 / \lambda_{0}$ is an eigenvalue of $R_{1} \mathcal{A}^{-1} \Longleftrightarrow \lambda_{0}$ is an eigenvalue of (1.16)-(1.17) $\Longleftrightarrow \lambda_{0}$ is an eigenvalue of (1.18)-(1.17) $\Longleftrightarrow\left[\begin{array}{c}z(0) \\ \gamma_{\lambda_{0}}(T) z(0)\end{array}\right] \in$ $\Lambda=\left[\begin{array}{l}X \\ Y\end{array}\right]$ for some $0 \neq z(0) \in \mathbb{C}^{2 n} \Longleftrightarrow\left(Y-\gamma_{\lambda_{0}}(T) X\right) a=0$ for some $0 \neq a \in \mathbb{C}^{2 n} \Longleftrightarrow$ $\operatorname{det}\left(Y-\gamma_{\lambda_{0}}(T) X\right)=0$, where we used Theorem 3.5 (c) in [27] for the first equivalence. where

ii) Note that $H: L_{R_{1}}^{2}\left([0, T] ; \mathbb{C}^{n}\right) \rightarrow L^{2}\left([0, T] ; \mathbb{C}^{n}\right), H u=R_{1}^{\frac{1}{2}} u$ defines an isometry,

$$
L_{R_{1}}^{2}\left([0, T] ; \mathbb{C}^{n}\right)=\left\{u \mid\|u\|_{R_{1}}^{2}:=\int_{0}^{T} u^{*} R_{1} u d t<\infty\right\} .
$$

Without confusion, we still use $R_{1}^{\frac{1}{2}}$ for the usual multiplication operator on $L^{2}\left([0, T] ; \mathbb{C}^{n}\right)$. Then

$$
\hat{\mathcal{A}}:=H^{-1} R_{1}^{-\frac{1}{2}} \mathcal{A} R_{1}^{-\frac{1}{2}} H
$$

defines a self-adjoint operator on $L_{R_{1}}^{2}\left([0, T] ; \mathbb{C}^{n}\right)$.

Denote the orders of $\operatorname{det}\left(I+\lambda R_{1} \mathcal{A}^{-1}\right)$ and $\operatorname{det}\left(Y-\gamma_{\lambda}(T) X\right)$ at the zero point $\lambda_{0}$ by $k_{0}$ and $k_{1}$, respectively. Let $\tau_{1}(\lambda, F)$ and $\tau_{2}(\lambda, F)$ be the algebraic and geometric multiplicities of $\lambda \in \sigma_{p}(F)$ for an operator $F$, respectively. Then it suffices to prove that for $i=1,2$,

$$
k_{0}=\tau_{i}\left(-1 / \lambda_{0}, R_{1} \mathcal{A}^{-1}\right)=\tau_{i}\left(-\lambda_{0}, R_{1}^{-\frac{1}{2}} \mathcal{A} R_{1}^{-\frac{1}{2}}\right)=\tau_{i}\left(-\lambda_{0}, \hat{\mathcal{A}}\right)=k_{1} .
$$

By Theorem 3.5(c) in [27] again,

$$
k_{0}=\tau_{1}\left(-1 / \lambda_{0}, R_{1} \mathcal{A}^{-1}\right)
$$

Since

$$
R_{1}^{-\frac{1}{2}}\left(R_{1} \mathcal{A}^{-1}\right) R_{1}^{\frac{1}{2}}=R_{1}^{\frac{1}{2}} \mathcal{A}^{-1} R_{1}^{\frac{1}{2}}
$$

we have $R_{1} \mathcal{A}^{-1}$ is diagonable and thus $-1 / \lambda_{0}$ is semi-simple, which means

$$
\tau_{1}\left(-1 / \lambda_{0}, R_{1} \mathcal{A}^{-1}\right)=\tau_{2}\left(-1 / \lambda_{0}, R_{1} \mathcal{A}^{-1}\right) .
$$

Note that $v$ is an eigenfunction of $-1 / \lambda_{0} \in \sigma\left(R_{1} \mathcal{A}^{-1}\right)$ if and only if $R_{1}^{\frac{1}{2}} \mathcal{A}^{-1} v$ is an eigenfunction of $-\lambda_{0} \in \sigma\left(R_{1}^{-\frac{1}{2}} \mathcal{A} R_{1}^{-\frac{1}{2}}\right)$. Thus,

$$
\tau_{2}\left(-1 / \lambda_{0}, R_{1} \mathcal{A}^{-1}\right)=\tau_{2}\left(-\lambda_{0}, R_{1}^{-\frac{1}{2}} \mathcal{A} R_{1}^{-\frac{1}{2}}\right) .
$$


Since $H$ is an isometry, and both $R_{1}^{-\frac{1}{2}} \mathcal{A} R_{1}^{-\frac{1}{2}}$ and $\hat{\mathcal{A}}$ are self-adjoint, we have

$$
\tau_{i}\left(-\lambda_{0}, R_{1}^{-\frac{1}{2}} \mathcal{A} R_{1}^{-\frac{1}{2}}\right)=\tau_{i}\left(-\lambda_{0}, \hat{\mathcal{A}}\right) \quad \text { for } i=1,2 \text {. }
$$

By Theorem 5.1 and Remark 5.1 in [11], we have $\tau_{i}\left(-\lambda_{0}, \hat{\mathcal{A}}\right)=k_{1}$ for $i=1,2 .^{\dagger}$ This proves (4.1).

Now, we are ready to prove Theorem 1.3: the Hill-type formula for Sturm-Liouville system (1.16)-(1.17).

Proof of Theorem 1.3. Since $R_{1} \mathcal{A}^{-1} \in \mathcal{J}_{1}$, by Theorem 3.7 in [27] we have

$$
\operatorname{det}\left(I+\lambda R_{1} \mathcal{A}^{-1}\right)=\prod_{j}\left(1-\lambda_{j}^{-1} \lambda\right) .
$$

First, we prove (1.20) for $R_{1}>0$. Following the proof of Lemma 2.3 in [17], we have $\gamma_{\lambda}(T)$ is an entire function on $\lambda$ and

$$
\left\|\gamma_{\lambda}(T)\right\| \leq C_{0}|\lambda|^{1 / 2} \exp \left(C|\lambda|^{1 / 2}\right)
$$

Then

$$
g(\lambda):=\operatorname{det}\left(Y-\gamma_{\lambda}(T) X\right)
$$

is an entire function and it is not hard to check that for any $\varepsilon>0$, there exists $C_{\varepsilon}$ such that

$$
|g(\lambda)| \leq C_{\varepsilon} \exp (\varepsilon|\lambda|) .
$$

By Lemma 4.1, all the zeros (counting orders) of $g(\lambda)$ are $\left\{\lambda_{j}\right\}$. Moreover, $\sum_{j}\left|\lambda_{j}\right|^{-1}<\infty$ due to $R_{1} \mathcal{A}^{-1} \in \mathcal{J}_{1}$. Since $\mathcal{A}$ is non-degenerate, $g(0) \neq 0$. By Lemma 3.6 in [27], we have

$$
g(0)^{-1} g(\lambda)=\prod_{j}\left(1-\lambda_{j}^{-1} \lambda\right)=\operatorname{det}\left(I+\lambda R_{1} \mathcal{A}^{-1}\right),
$$

and in particular,

$$
\prod_{j}\left(1-\lambda_{j}^{-1}\right)=g(0)^{-1} g(1)
$$

which proves (1.20) for $R_{1}>0$. In the general case, we choose $\alpha_{0} \in \mathbb{R}$ such that $R_{1}-\alpha_{0}>$ 0 and $\mathcal{A}+\alpha_{0}$ is non-degenerate. We rewrite $\gamma_{\lambda}(T)$ by $\gamma_{\lambda}\left(T, R_{1}\right)$ to indicate its dependence on $R_{1}$. Following (2.5) in [17], we have

$$
\begin{aligned}
& \operatorname{det}\left(I+R_{1} \mathcal{A}^{-1}\right)=\operatorname{det}\left[I+\left(R_{1}-\alpha_{0}\right)\left(\mathcal{A}+\alpha_{0}\right)^{-1}\right] \cdot \operatorname{det}\left(I+\alpha_{0} \mathcal{A}^{-1}\right) \\
= & {\left[\operatorname{det}\left(Y-\gamma_{\alpha_{0}}\left(T, I_{n}\right) X\right)^{-1} g(1)\right] \cdot\left[g(0)^{-1} \operatorname{det}\left(Y-\gamma_{\alpha_{0}}\left(T, I_{n}\right) X\right)\right]=g(0)^{-1} g(1), }
\end{aligned}
$$

where we used (4.2). This proves (1.20) in the general case.

${ }^{\dagger}$ Although the Sturm-Liouville system (1.16) is slightly general than (1.1) in [11] due to the additional ' $Q$ 'terms, the same proof of Theorem 5.1 in [11] works here. 
Next, we give the proof of Theorem 1.4.

Proof of Theorem 1.4. Since $R_{1} \mathcal{A}^{-1} \in \mathcal{J}_{1}$, we have by (5.12) in [27] that

$$
\operatorname{det}\left(I+\lambda R_{1} \mathcal{A}^{-1}\right)=\exp \left(\sum_{m=1}^{\infty} \frac{(-1)^{m+1}}{m} \lambda^{m} \operatorname{Tr}\left(\left(R_{1} \mathcal{A}^{-1}\right)^{m}\right)\right) .
$$

Let

$$
f(\lambda)=\operatorname{det}\left(Y-\gamma_{\lambda}(T) X\right) \cdot \operatorname{det}\left(Y-\gamma_{0}(T) X\right)^{-1} .
$$

Similar to (3.12), we have $f(\lambda)=e^{h(\lambda)}$, where

$$
\frac{h^{(m)}(0)}{m !}=\sum_{k=1}^{m} \frac{-1}{k}\left(\sum_{j_{1}+\cdots+j_{k}=m} \operatorname{Tr}\left(G_{j_{1}} \cdots G_{j_{k}}\right)\right) .
$$

Here $G_{j}=P^{-1} M_{j} X, M_{j}$ and $\hat{D}(t)$ are defined in (1.11), $D(t)$ is defined in (1.19). By Theorem 1.3, we get the parameterized Hill-type formula $\operatorname{det}\left(I+\lambda R_{1} \mathcal{A}^{-1}\right)=f(\lambda)$. Comparing the coefficients of (4.3) and (4.4), we have

$$
\begin{aligned}
& \sum_{k=1}^{m} \frac{1}{k}\left(\sum_{j_{1}+\cdots+j_{k}=m} \operatorname{Tr}\left(G_{j_{1}} \cdots G_{j_{k}}\right)\right)=\frac{(-1)^{m}}{m} \operatorname{Tr}\left(\left(R_{1} \mathcal{A}^{-1}\right)^{m}\right) \\
= & \frac{(-1)^{m}}{m} \sum_{j} \frac{(-1)^{m}}{\lambda_{j}^{m}}=\frac{1}{m} \sum_{j} \frac{1}{\lambda_{j}^{m}} .
\end{aligned}
$$

This complete the proof.

Moreover, we have

$$
\operatorname{Tr}\left(R_{1} \mathcal{A}^{-1}\right)^{k}=\operatorname{Tr}\left(\mathcal{F}^{k}(B, D)\right), \quad k \in \mathbb{N},
$$

where $B$ and $D$ are defined in (1.18).

When the Hamiltonian system comes from the Legendre transformation of SturmLiouville system, the operator $\mathcal{F}(B, D) \in \mathcal{J}_{\infty}$, and thus,

$$
\operatorname{det}(I-\mathcal{F})=\prod_{j}\left(1-\lambda_{j}^{-1}\right) \quad \text { and } \quad \operatorname{Tr}(\mathcal{F})=\sum_{j} \lambda_{j}^{-1} .
$$

(4.5) does not hold for general Hamiltonian systems, and a counterexample could be found in Subsection 3.3 of [13].

Finally, we give an example to illustrate how to get infinite identity from the trace formula. Consider the eigenvalue problem

$$
\ddot{y}+\lambda y=0
$$


with the boundary conditions

$$
y(0)=0 \quad \text { and } \quad \cos (\theta) y(T)+\sin (\theta) \dot{y}(T)=0, \quad \theta \in[0, \pi / 2] .
$$

It is well known that the $k$-th eigenvalue $\lambda_{k}$ is the $k$-th positive solution of the next transcendental equation

$$
\tan (\sqrt{\lambda} T)=-\tan (\theta) \sqrt{\lambda}
$$

It is easy to check that if $\theta=0$, then $\lambda_{k}=\frac{k^{2} \pi^{2}}{T^{2}}$; and if $\theta=\pi / 2$, then $\lambda_{k}=\frac{\pi^{2}}{T^{2}}\left(k-\frac{1}{2}\right)^{2}$. For $\theta \in(0, \pi / 2)$, it is obvious that

$$
\left(k-\frac{1}{2}\right) \pi<\sqrt{\lambda_{k}} T<k \pi .
$$

However, $\lambda_{k}$ can only be solved numerically. As an application of the trace formula, we have the following equality, which itself is interesting:

$$
\sum_{k \in \mathbb{N}} \frac{1}{\lambda_{k}}=\frac{3 T^{2} \sin (\theta)+T^{3} \cos (\theta)}{6(\sin (\theta)+T \cos (\theta))} .
$$

Obviously, for $\theta=0$, (4.9) gives the well known identity

$$
\sum_{k \in \mathbb{N}} \frac{1}{k^{2}}=\frac{\pi^{2}}{6}
$$

and for $\theta=\pi / 2,(4.9)$ gives the identity

$$
\sum_{k \in \mathbb{N}} \frac{1}{\pi^{2}\left(k-\frac{1}{2}\right)^{2}}=\frac{1}{2}
$$

To the best of our knowledge, for $\theta \in(0, \pi / 2)$, we don't know any such kind of formula on the sum of $\frac{1}{\lambda_{k}}$ ever before. The detailed calculation could be found in [17].

\section{Applications}

The relative Morse index for linear Hamiltonian system is equal to the Maslov-type index for the corresponding fundamental solutions, and the Maslov-type index is a very useful tool in studying the multiplicity and stability of periodic solution in Hamiltonian systems [22]. In this section, we give the relation of conditional Fredholm determinant and relative Morse index, also we estimate the relative Morse index by the trace formula. Therefore the trace formula could be used to judge the linear stability via the Maslov-type index. 


\subsection{Relation with the relative Morse index and Maslov-type index}

A simple way to understand the relative Morse index $I(A-B, A-B-D)$ is from the viewpoint of spectral flow. For reader's convenience, we first give a brief review of the spectral flow. The spectral flow was introduced by Atiyah, Patodi and Singer [1] in their study of index theory on manifolds with boundary. Let $\{A(\theta), \theta \in[0,1]\}$ be a continuous path of self-adjoint Fredholm operators on a Hilbert space $\mathcal{H}$. Roughly speaking, the spectral flow of path $\{A(\theta), \theta \in[0,1]\}$ counts the net change in the number of negative eigenvalues of $A(\theta)$ as $\theta$ goes from 0 to 1 , where the enumeration follows from the rule that each negative eigenvalue crossing to the positive axis contributes +1 and each positive eigenvalue crossing to the negative axis contributes -1 , and for each crossing, the multiplicity of eigenvalue is counted.

Our main interests in this paper are Hamiltonian dynamics. Given a Hamiltonian system

$$
\dot{z}=J H^{\prime}(t, z),
$$

we consider its solution under some self-adjoint boundary condition. Let $z$ be a solution to (5.1), and the fundamental solution of the linearized Hamiltonian system be $\gamma:[0, T] \rightarrow \operatorname{Sp}(2 n)$ with $\gamma(0)=I_{2 n}$. The corresponding linear operator is $-J \frac{d}{d t}-B(t)$ with $B(t)=H^{\prime \prime}(t, z(t))$. Let $E_{\Lambda}$ be defined in (1.6) for $\Lambda \in \operatorname{Lag}(\mathcal{V}, \Omega)$. Then both $-J \frac{d}{d t}$ and $-J \frac{d}{d t}-B(t)$ are self-adjoint Fredholm operators with domain $E_{\Lambda}$.

In general, suppose

$$
B_{s}(t)=B(s, t) \in C([0,1] \times[0, T] ; \mathcal{S}(2 n)) .
$$

Let $B_{s} \in C([0, T] ; \mathcal{S}(2 n))$ for $s \in[0,1]$. Then each operator $-J \frac{d}{d t}-B_{s}(t)$ has domain $E_{\Lambda}$. For such two operators $A-B_{0}$ and $A-B_{1}$, we can define the relative Morse index via spectral flow. In fact, by [14] we have

$$
\mathcal{I}\left(A-B_{0}, A-B_{1}\right)=-S f(\{A-B(s), s \in[0,1]\}) .
$$

There is another natural topological characterization for each orbit of the system, namely its Maslov index [4]. Here we use the Maslov index theory of the complex Lagrangian subspaces, the details of which can be found in [29]. Let $\left(\mathbb{C}^{2 n}, \omega_{n}\right)$ be the complex symplectic vector space. Recall that a complex subspace $V$ is Lagrangian if $\left.\omega_{n}\right|_{V}=0$ and $\operatorname{dim}_{C} V=n$. Let $V^{ \pm}=\operatorname{ker}\left(i J \mp I_{2 n}\right)$. Then any Lagrangian subspace can be expressed as $\operatorname{Gr}(U)=\left\{(x, U x) \mid x \in V^{+}\right\}$, where $U: V^{+} \rightarrow V^{-}$is some unitary matrix, and the converse is also true. This shows that the (complex) Lagrangian Grassmannian $\operatorname{Lag}\left(\mathbb{C}^{2 n}\right)$ is homeomorphic (isomorphic) to the unitary group $U(n)$, which we denote by $F: \operatorname{Lag}\left(\mathbb{C}^{2 n}\right) \rightarrow U(n)$. For $V_{1}, V_{2} \in \operatorname{Lag}\left(\mathbb{C}^{2 n}\right)$, it is obvious that

$$
\operatorname{dim}\left(V_{1} \cap V_{2}\right)=\operatorname{dim} \operatorname{ker}\left(F\left(V_{2}\right)^{-1} F\left(V_{1}\right)-I_{n}\right) .
$$


For any fixed $U \in U(n)$, let

$$
\left.\Sigma_{U}=\left\{U_{0} \in U(n) \mid \operatorname{det}\left(U^{-1} U_{0}-I_{n}\right)=0\right)\right\}
$$

be the singular cycle of $U$. For any $U_{0} \in \Sigma_{U}$, the path $e^{i t} U_{0}(|t|<\varepsilon)$ is transversal to $\Sigma_{U}$. Let $U(t)(t \in[a, b])$ be any path in $U(n)$. For $\varepsilon>0$ small enough, $e^{-\varepsilon i} U(a)$ and $e^{-\varepsilon i} U(b)$ are not in the singular cycle of $U$, and the intersection number $\left[e^{-\varepsilon i} U(t): \Sigma_{U}\right]$ is well defined. For a path of complex Lagrangian subspaces $V(t)$ and $\Lambda \in \operatorname{Lag}\left(\mathbb{C}^{2 n}\right)$, we define the Maslov index to be

$$
\mu(\Lambda, V(t)):=\left[e^{-\varepsilon i} \mathcal{F}(V(t)): \Sigma_{\mathcal{F}(\Lambda)}\right] .
$$

For the fundamental solution $\gamma(t), t \in[0, T]$ of the linearized Hamiltonian system along the solution $z(t)$, the Maslov index of $z$ is defined to be

$$
\mu(z)=\mu(\Lambda, G r(\gamma(t))),
$$

where $\Lambda \in \operatorname{Lag}(\mathcal{V}, \Omega)$ is the boundary condition.

Theorem 5.1. In [14], the authors showed that for each orbit of the Hamiltonian system, its relative Morse index is equal to its Maslov index under the Lagrangian boundary conditions, that is,

$$
I\left(-J \frac{d}{d t},-J \frac{d}{d t}-B(t)\right)=\mu(\Lambda, G r(\gamma(t))) .
$$

The Maslov-type index for symplectic paths is a powerful tool in studying the stability problem. We give its relation to the Maslov index, and the details can be found in $[22,23]$. As usual, consider paths in $\operatorname{Sp}(2 n)$ :

$$
\mathcal{P}_{T}(2 n)=\left\{\gamma \in C([0, T] ; \operatorname{Sp}(2 n)) \mid \gamma(0)=I_{2 n}\right\} .
$$

For any $\omega \in \mathbb{U}$ and $\gamma \in \mathcal{P}_{T}(2 n)$, we define

$$
\begin{aligned}
& v_{\omega}(\gamma)=\operatorname{dim}_{\mathbb{C}} \operatorname{ker}_{\mathbb{C}}\left(\gamma(T)-\omega I_{2 n}\right), \\
& i_{\omega}(\gamma)=\left[e^{-\varepsilon J} \gamma * \xi_{n}: \operatorname{Sp}(2 n)_{\omega}^{0}\right],
\end{aligned}
$$

the intersection number with $\varepsilon$ to be a small positive number, where

$$
\operatorname{Sp}(2 n)_{\omega}^{0}=\left\{M \in \operatorname{Sp}(2 n) \mid D_{\omega}(M)=0\right\}
$$

is the codimensional one hypersurface in $\mathrm{Sp}(2 n)$ with

$$
D_{\omega}(M)=(-1)^{n-1} \bar{\omega}^{n} \operatorname{det}\left(M-\omega I_{2 n}\right), \quad \forall \omega \in \mathbb{U}, \quad M \in \operatorname{Sp}(2 n) .
$$

Then we have the following theorem, which is from [23]. 
Theorem 5.2. For any $\gamma \in \mathcal{P}_{T}(2 n)$, we have

$$
\begin{aligned}
& i_{1}(\gamma)+n=\mu(\triangle, G r(\gamma(t))), \\
& i_{\omega}(\gamma)=\mu(\operatorname{Gr}(\omega), \operatorname{Gr}(\gamma(t))), \quad \omega \in \mathbb{U} \backslash\{1\},
\end{aligned}
$$

where $\triangle$ is the diagonal $G r\left(I_{2 n}\right)$ and $G r(\omega)=G r\left(\omega I_{2 n}\right)$.

Now, we briefly review the Morse index theorem of Sturm-Liouville systems with any self-adjoint boundary condition, and the details can be found in [18]. Consider the Sturm-Liouville system (1.16). We define the Morse index of Sturm-Liouville operator by $m^{-}(\mathcal{A})$ which is the number of total negative eigenvalues of $\mathcal{A}$, and set $m^{0}(\mathcal{A})=\operatorname{ker}(\mathcal{A})$. Then we have the following Morse index theorem from [18], which gives the relation between the Morse index of $\mathcal{A}$ and the Maslov index of the corresponding Hamiltonian system.

Theorem 5.3. For the Sturm-Liouville system (1.16) under the boundary condition (1.17), we have

$$
\mu(\Lambda, G r(\gamma(t)), t \in[0, T])-m^{-}(\mathcal{A})=n-i\left(G r\left(I_{2 n}\right), \Lambda, \Lambda_{D}\right),
$$

where $i\left(\operatorname{Gr}\left(I_{2 n}\right), \Lambda, \Lambda_{D}\right)$ is the Duistermaat triple index.

The details of triple index can be found in $[8,28]$. Let $\Lambda_{N}^{n}=\{0\} \oplus \mathbb{C}^{n}$ and $\Lambda_{D}^{n}=\mathbb{C}^{n} \oplus$ $\{0\}$, which are Lagrangian subspaces of $\left(\mathbb{C}^{2}, \omega_{n}\right)$ and can be considered as the Neumann and Dirichlet boundary conditions, respectively. We set $\Lambda_{D}=\Lambda_{D}^{n} \oplus \Lambda_{D}^{n}$ and $\Lambda_{N}=$ $\Lambda_{N}^{n} \oplus \Lambda_{N}^{n}$. It is evident that

$$
\begin{aligned}
& \Lambda_{D}=\left\{(z(0), z(T)) \in \mathbb{C}^{4 n} \mid y(0)=y(T)=0\right\} \\
& \left\{(z(0), z(T)) \in \mathbb{C}^{4 n} \mid x(0)=x(T)=0\right\}
\end{aligned}
$$

We list several important examples to compute $i\left(G r\left(I_{2 n}\right), \Lambda, \Lambda_{D}\right)$.

1. The first is from Theorem 1.2 in [14]. Let $V$ be any subspace of $\Lambda_{N}$ and the boundary condition be given by $\Lambda_{V}=\mathcal{J} V^{\perp} \oplus V$, where $\mathcal{J}=-J_{n} \oplus J_{n}$. Then

$$
i\left(G r\left(I_{2 n}\right), \Lambda, \Lambda_{D}\right)=n-\operatorname{dim}\left(V^{\perp} \cap G r\left(-I_{n}\right)\right) .
$$

There are two important cases:

(i) $\operatorname{dim}\left(V^{\perp} \cap G r\left(-I_{n}\right)\right)=\operatorname{dim}\left(M-I_{2 n}\right), \forall V=G r(M), M \in G L\left(\mathbb{C}^{n}\right)$. Let $V_{1}$ and $V_{2}$ be two subspaces of $\mathbb{C}^{n}$. Then

(ii) $\operatorname{dim}\left(V^{\perp} \cap \operatorname{Gr}\left(-I_{n}\right)\right)=\operatorname{dim}\left(V_{1}^{\perp}\right) \cap V_{2}^{\perp}, \forall V=V_{1} \oplus V_{2}$. As special cases, we have

a. Dirichlet boundary condition: $i\left(G r\left(I_{2 n}\right), \Lambda_{D}, \Lambda_{D}\right)=0$.

b. Neumann boundary condition: $i\left(G r\left(I_{2 n}\right), \Lambda_{N}, \Lambda_{D}\right)=n$. 
c. Periodic boundary condition: $i\left(G r\left(I_{2 n}\right), G\left(I_{2 n}\right), \Lambda_{D}\right)=0$.

2. Separated boundary conditions. For this case, we have

$$
i\left(G r\left(I_{2 n}\right), \Lambda_{s} \oplus \Lambda_{e}, \Lambda_{D}\right)=n-\operatorname{dim}\left(\Lambda_{s} \cap \Lambda_{D}^{n}\right)+i\left(\Lambda_{s}, \Lambda_{e}, \Lambda_{D}^{n}\right) .
$$

For the special case $y(0)=A_{s} x(0), y(T)=A_{e} x(T)$, we have

$$
i\left(G r\left(I_{2 n}\right), \Lambda_{s} \oplus \Lambda_{e}, \Lambda_{D}\right)=n+m^{+}\left(A_{s}-A_{e}\right),
$$

where $m^{+}(A)$ is the total number of positive eigenvalues of operator $A$.

\subsection{Estimation of the relative Morse index and the stability criteria}

In this subsection, we consider the relation of conditional Fredholm determinant and relative Morse index, also we could estimate the relative Morse index by the Krein-type trace formula. As in $[12,15]$, we have the following theorem.

Theorem 5.4. Assume $A-B$ and $A-B-D$ are non-degenerate, then $\operatorname{det}(I-\mathcal{F}(B, D))>0$ $(<0)$ if and only if $\mathcal{I}(A-B, A-B-D)$ is even (odd).

In [12], the trace of $\mathcal{F}^{k}(B, D)$ was used to get the nontrivial estimation of relative Morse index. Although we dealt with operators in the S-periodic case in [12], it is totally same for the Lagrangian boundary conditions. The following theorem is from [12].

Proposition 5.1. Suppose $A-B$ is non-degenerate and $D>0$. Then for $k \in \mathbb{N}$,

$$
I(A-B, A-B-D)+v(A-B-D)<\operatorname{Tr}\left(\mathcal{F}^{2 k}\right),
$$

where $v(A-B-D)=\operatorname{dim} \operatorname{ker}(A-B-D)$.

Proposition 5.2. Suppose $D>0$. Then

$$
-\operatorname{Tr}\left(\mathcal{F}^{2 k}\right)<I(A-B, A-B+D) \leq 0, \quad \forall k \in \mathbb{N} .
$$

Corollary 5.1. Suppose $D>0$. If $\operatorname{Tr}\left(\mathcal{F}^{2 k}\right) \leq 1$ for some $k \in \mathbb{N}$, then

$$
I(A-B, A-B+D)=I(A-B, A-B-D)+v(A-B-D)=0 .
$$

Theorem 5.5. Suppose $A-B$ is non-degenerate. Assume that there exist $D_{1}, D_{2} \in \mathcal{B}(2 n)$ such that $D_{1}<D<D_{2}$ with $D_{1}<0, D_{2}>0$. If there exists $k \in 2 \mathbb{N}$ such that $\operatorname{Tr}^{k}\left(B, D_{j}\right)<1$ for $j=1,2$, then $A-B-D$ is non-degenerate, and moreover, $\mathcal{I}(A-B, A-B-D)=0$.

Theorem 5.6. Suppose $A-B$ is non-degenerate and $D_{1} \leq D \leq D_{2}$, where $D_{1}<0, D_{2}>0$. Let

$$
m^{-}=\inf \left\{\left[\operatorname{Tr}\left(\mathcal{F}\left(B, D_{1}\right)^{k}\right)\right], k \in 2 \mathbb{N}\right\} \quad \text { and } \quad m^{+}=\inf \left\{\left[\operatorname{Tr}\left(\mathcal{F}\left(B, D_{2}\right)^{k}\right)\right], k \in 2 \mathbb{N}\right\} .
$$

Then

$$
-m^{-} \leq I(A-B, A-B-D) \leq m^{+} .
$$


Connected with the trace formula (1.12), we can give an estimation of relative Morse index by the trace of matrices. As a corollary of Theorem 5.5, we have

Corollary 5.2. Suppose $A-B$ is non-degenerate. Assume that $D \geq 0, \operatorname{Tr}\left(G_{1}^{2}\right)+2 \operatorname{Tr}\left(G_{2}\right)<1$. Then $A-B-D$ is non-degenerate, and $\mathcal{I}(A-B, A-B-D)=0$. The case $D<0$ is similar.

Now we give the stability criteria. Suppose $z(t)$ is a $T$-periodic solution of Hamiltonian system (5.1) with the fundamental solutions $\gamma(t) . z$ is called (spectrally) stable if $\sigma(\gamma(T)) \subset \mathbb{U}$, is called hyperbolic if $\sigma(\gamma(T)) \cap \mathbb{U}=\varnothing$. we denote $\tilde{\gamma}$ to be the fundamental solutions with respect to $B+D$, and write $\widetilde{M}=\tilde{\gamma}(T)$. In many physical systems, $D$ can be regarded as the perturbation of the original system with respect to $B$. It is natural to consider the problems: can we give some criteria such that the original system is stable (or hyperbolic)? How can we estimate the perturbation region such that its stability (or hyperbolicity) still preserves? Our trace formula is a useful tool to study these problems. In order to estimate the stability, we use the Maslov-type index $i_{\omega}(\gamma)$ as mentioned above.

Let $e(M)$ be the total number of eigenvalues of $M$ on $\mathbb{U}$. Then a simple but useful stability criterion is

$$
e(\gamma(T)) / 2 \geq\left|i_{-1}(\gamma)-i_{1}(\gamma)\right| .
$$

Then we have following propositions.

Proposition 5.3. Let $\omega=e^{v T}, v \in \sqrt{-1} \mathbb{R}$ and consider the boundary condition $\Lambda=$ $\operatorname{Gr}\left(\omega I_{2 n}\right)$. Suppose $D_{1} \leq D \leq D_{2}$, where $D_{1}<0, D_{2}>0$. If

$$
\operatorname{Tr}\left(\mathcal{F}\left(B, D_{j}\right)^{2}\right) \leq 1, \quad j=1,2,
$$

hold true for any $v \in \sqrt{-1} \mathbb{R}$, then $i_{\omega}(\gamma)=i_{\omega}(\tilde{\gamma})$. Especially, if $\operatorname{Tr}\left(\mathcal{F}\left(B, D_{j}\right)\right) \leq 1$ for $v=0, v=\sqrt{-1} \pi / T$ and $j=1,2$, then

$$
e(\widetilde{M}) / 2 \geq\left|i_{1}(\gamma)-i_{-1}(\gamma)\right| .
$$

If $\left|i_{1}(\gamma)-i_{-1}(\gamma)\right|=n$ for the original system, then after the perturbation by $D$, we still have $e(\widetilde{M})=2 n$, and thus it remains spectrally stable. We can compute the trace by the trace formula given by (1.12), hence the trace formula can estimate the stable region of the perturbation system.

Proposition 5.4. Suppose $M$ is hyperbolic and consider the boundary condition $\Lambda=G r\left(\omega I_{2 n}\right)$ for $\omega=e^{v T}, v \in \sqrt{-1} \mathbb{R}$. If

$$
\operatorname{Tr}\left(\mathcal{F}^{2}((B, D))\right) \leq 1 \quad \text { for } \quad v \in\left[0, \frac{\sqrt{-1} \pi}{T}\right]
$$

then $\widetilde{M}$ is hyperbolic.

For the applications of the trace formula to stability estimation in the planar threebody problem, we refer the readers to $[12,13]$ for the details. 


\section{Acknowledgements}

The first author is partially supported by NSFC (Nos. 12071255 and 11790271) and National Key R\&D Program of China (2020YFA0713300). The second authors is partially supported by NSFC (No. 11801583). The third author is Partially supported by NSFC (Nos. 11471189, and 11871308).

\section{References}

[1] M. F. Atiyah, V. K. Patodi and I. M. Singer, Spectral asymmetry and Riemannian geometry. III, Math. Proc. Cambridge Philos. Soc., 79 (1976), 71-99.

[2] S. V. Bolotin, The Hill determinant of a periodic orbit, Mosc. Univ. Mech. Bull., 43(3) (1988), $7-11$.

[3] S. V. Bolotin and D. V. Treschev, Hill's formula, Russian Math. Surveys, 65 (2010), 191-257.

[4] S. E. Cappell, R. Lee and E. Y. Miller, On the Maslov index, Commun. Pure Appl. Math., 47 (1994), 121-186.

[5] M. Davletshin, Hill formula for $g$-periodic trajectories of Lagrangian systems, Trudy MMO, 74 (2013), Trans. Moscow Math. Soc. Tom, 74 (2013), 65-96.

[6] R. Denk, On Hilbert-Schmidt operators and determinants corresponding to periodic ODE systems, Differential and integral operators (Regensburg, 1995), 57-71, Oper. Theory Adv. Appl., Vol. 102, Birkhäuser, Basel, 1998.

[7] T. Dreyfuss and H. Dym, Product formulas for the eigenvalues of a class of boundary value problems, Duke Math. J., 45 (1978), 15-37.

[8] J. J. Duistermaat, On the Morse index in variational calculus, Adv. Math., 21 (1976), 173-195.

[9] R. Forman, Functional determinants and geometry, Invent. Math., 88 (1987), 447-493.

[10] G. W. Hill, On the part of the motion of the lunar perigee which is a function of the mean motions of the sun and moon, Cambridge, Wilson, 1877; reprinted with some additions at Acta Math., 8 (1886), 1-36.

[11] X. Hu, L. Liu, L. Wu and H. Zhu, Singularity of the $n$-th eigenvalue of high dimensional Sturm-Liouville problems, J. Differential Equations, 266 (2019), 4106-4136.

[12] X. Hu, Y. Ou and P. Wang, Trace Formula for Linear Hamiltonian Systems with its Applications to Elliptic Lagrangian Solutions, Arch. Ration. Mech. Anal., 216 (2015), 313-357.

[13] X. Hu, Y. Ou and P. Wang, Hill-type formula for Hamiltonian system with Lagrangian boundary conditions, J. Differential Equations, 267 (2019), 2416-2447.

[14] X. Hu and S. Sun, Index and stability of symmetric periodic orbits in Hamiltonian systems with its application to figure-eight orbit, Commun. Math. Phys., 290 (2009), 737-777.

[15] X. Hu and P. Wang, Conditional Fredholm determinant of $S$-periodic orbits in Hamiltonian systems, J. Funct. Anal., 261 (2011), 3247-3278.

[16] X. Hu and P. Wang, Hill-type formula and Krein-type trace formula for S-periodic solutions in ODEs, Discrete Contin. Dyn. Syst., 36 (2016), 763-784.

[17] X. Hu and P. Wang, Eigenvalue problem of Sturm-Liouville systems with separated boundary conditions, Math. Z., 283 (2016), 339-348.

[18] X. Hu, L. Wu and R. Yang, Morse index theorem of Lagrangian systems and stability of brake orbit, J. Dynam. Differential Equations, 32 (2020), 61-84.

[19] M. G. Krein, On tests for the stable boundedness of solutions of periodic canonical systems, PrikL Mat. Mekh., 19 (1955), 641-680. 
[20] M. G. Krein, Foundation of the theory of $\lambda$-zones of stability of a canonical systems of linear differential equations with periodic coefficients, In Memoriam: A. A. Andronov, Izdat. Akad. Nauk SSSR, Moscow, (1955), 413-498.

[21] S. Levit and U. Smilansky, A theorem on infinite products of eigenvalues of Sturm-Liouville type operators, Proc. Amer. Math. Soc., 65 (1977), 299-302.

[22] Y. Long, Index Theory for Symplectic Paths with Applications, Progress in Math., 207, Birkhäuser, Basel, 2002.

[23] Y. Long and C. Zhu, Maslov-type index theory for symplectic paths and spectral flow, II, Chinese Ann. Math. Ser. B, 21 (2000), 89-108.

[24] H. Poincaré, Sur les déterminants d'ordre infini, Bull. Soc. Math. France, 14 (1886), 77-90.

[25] A. Portaluri and L. Wu, Spectral flow, Brouwer degree and Hill's determinant formula, J. Differential Equations, 269 (2020), 7253-7286.

[26] D. B. Ray and I. M. Singer, R-Torsion and the Laplacian on Riemann manifolds, Adv. Math., 7 (1971), 145-210.

[27] B. Simon, Trace Ideals and Their Applications, Second edition, Mathematical Surveys and Monographs, 120. American Mathematical Society, Providence, RI, 2005.

[28] Y. Zhou, L. Wu and C. Zhu, Hörmander index in finite-dimensional case, Front. Math. China, 13 (2018), 725-761.

[29] C. Zhu, A generalized Morse Index Theorem, in: Analysis, Geometry and Topology of Elliptic Operators, Hackensack, NJ: World Sci. Publ., 2006, 493-540. 